\title{
Incidencia de bacterias multi-resistentes en unidades de cuidados intensivos de hospitales chilenos
}

\author{
M. Paz Acuña, Marcela Cifuentes, Francisco Silva, Álvaro Rojas, Jaime Cerda y Jaime Labarca \\ en representación del Grupo Colaborativo de Resistencia Bacteriana (GCRB) de Chile*
}

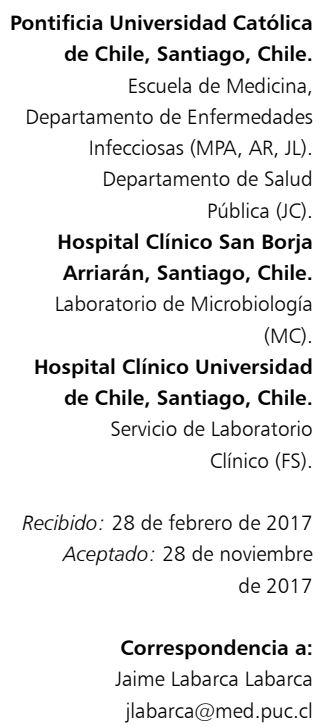

Pontificia Universidad Católica de Chile, Santiago, Chile. Escuela de Medicina, Departamento de Enfermedades Infecciosas (MPA, AR, JL). Departamento de Salud Pública (JC). Hospital Clínico San Borja Arriarán, Santiago, Chile. Laboratorio de Microbiología

Hospital Clínico Universidad de Chile, Santiago, Chile. Servicio de Laboratorio Clínico (FS)

Recibido: 28 de febrero de 2017 Aceptado: 28 de noviembre de 2017

Correspondencia a: Jaime Labarca Labarca jlabarca@med.puc.cl

\section{Incidence of multi-resistant bacteria in Intensive Care Units of Chilean hospitals}

Introduction: Incidence of multi-resistant bacteria is an indicator that permits better estimation of the magnitude of bacterial resistance in hospitals. Aim: To evaluate the incidence of relevant multi-drug resistant bacteria in intensive care units (ICUs) of Chile. Methods: Participating hospitals submitted information about the number of isolates from infected or colonized patients with 7 epidemiologically relevant multi-resistant bacteria in adult and pediatric ICUs between January 1, 2014 and October 31, 2015 and the number of bed days occupied in these units in the same period was requested. With these data incidence was calculated per 1,000 patient days for each unit. Results: Information from 20 adults and 9 pediatric ICUs was reviewed. In adult ICUs the bacteria with the highest incidence were $K$. pneumoniae ESBL [4.72 x 1,000 patient day (1.21-13.89)] and oxacillin -resistant $S$. aureus [3.85 (0.71-12.66)]. In the pediatric units the incidence was lower, highlighting $K$. pneumoniae ESBL [2.71 (0-7.11)] and carbapenem -resistant $P$. aeruginosa $[1.61$ (0.31-9.25)]. Conclusion: Importantdifferences between hospitals in the incidence of these bacteria were observed. Incidence of multi-resistant bacteria in adult ICU was significantly higher than in pediatric ICU for most of the studied bacterias.

Key words: Bacterial resistance, ICU, multiple drug resistance, incidence.

Palabras clave: Resistencia bacteriana, UCI, bacterias multi-resistentes, incidencia.

\section{Introducción}

L a resistencia bacteriana constituye un problema relevante de salud pública que ha cobrado cada vez más importancia, tanto por un aumento en su incidencia, como por la aparición de nuevos mecanismos de resistencia que limitan las alternativas terapéuticas. Esto tiene especial repercusión en unidades de cuidados intensivos, que concentran la mayor incidencia de bacterias multi-resistentes y las infecciones clínicas de mayor gravedad, lo que determina un mayor consumo de antimicrobianos, como han evidenciado estudios multicéntricos recientes realizados en Chile ${ }^{1-2}$.

La vigilancia de bacterias multi-resistentes específicas, definidas por su relevancia epidemiológica, es esencial para evaluar la magnitud del problema de la resistencia bacteriana. En este contexto, el Grupo Colaborativo de
Resistencia Bacteriana (GCRB) de la Sociedad Chilena de Infectología, ha trabajado en la consolidación de datos de resistencia a nivel nacional y su difusión a través de distintas publicaciones. A la fecha, se han publicado los datos de susceptibilidad de microorganismos relevantes en los años $2009^{3}$ y $2012^{4}$, donde se ha evaluado el porcentaje de susceptibilidad para múltiples antimicrobianos en determinadas bacterias provenientes de muestras hospitalarias recibidas por los laboratorios. Complementariamente, tener la información de incidencia de bacterias multiresistentes, que ajusta la información a un denominador común (pacientes-día), permite estimar mejor la magnitud del problema epidemiológico de resistencia, establecer comparaciones entre distintos servicios clínicos y entre distintos hospitales, y también evaluar comparativamente su evolución en el tiempo.

El objetivo de este estudio fue determinar incidencia

*Grupo Colaborativo de Resistencia Bacteriana de Chile**: Hospital Dr. Carlos Van Buren, Valparaíso (Gerardo Peralta, Gonzalo Wilson). Hospital Dr. Eduardo Pereira, Valparaíso (Rubén Muñoz). Clínica Reñaca, Viña del Mar (Luis Bavestrello). Hospital Regional de Rancagua (Andrés Cornejo). Hospital Regional de Temuco Dr. Hernán Henríquez (Vijna Illesca). Hospital Clínico Regional de Valdivia, Valdivia (M. Carolina Cruz). Hospital Puerto Montt, Puerto Montt (M. Luisa Rioseco). Hospital Clínico Magallanes Dr. Lautaro Navarro Avaria, Punta Arenas (Rodrigo Muñoz). Hospital de la Fuerza Aérea General Dr. Raúl Yazigi J, Santiago (Erna Cona). Hospital DIPRECA, Santiago (Margareta Mühlhauser). Hospital Clínico Universidad de Chile, Santiago (Francisco Silva). Hospital Clínico Pontificia Universidad Católica de Chile, Santiago (Paulette Legarraga). Hospital Dr. Exequiel González Cortés, Santiago (Ernesto Paya). Hospital Clínico de Niños Roberto del Río, Santiago (Dona Benadof). Hospital Del Salvador, Santiago (Luz María Fuenzalida). Hospital San José, Santiago (Maggie Vecchiola), Hospital Clínico San Borja Arriarán, Santiago (Marcela Cifuentes). Hospital Padre Hurtado, Santiago (Pamela Rojas). Hospital San Juan de Dios, Santiago (Alejandra Céspedes, Leonardo Chanqueo). Clínica Alemana, Santiago (Lorena Porte). Clínica Las Condes, Santiago (Beatrice Hervé). Clínica Dávila, Santiago (Cecilia Tapia).

**Enumeración según orden geográfico de las instituciones (de norte a sur del país). 
de bacterias multi-resistentes epidemiológicamente relevantes en unidades de cuidados intensivos de adultos y pediátricos de Chile y establecer un consolidado nacional con medianas, percentiles 25-75 y rangos de dispersión que permitan comparar datos de cada hospital con este referente nacional. Además, se busca determinar si existen diferencias significativas en la incidencia entre población adulta y pediátrica.

\section{Materiales y Método}

Se invitó a participar a hospitales y clínicas de la red que integra el GCRB. Se les solicitó vía correo electrónico información del período comprendido entre el 01 de enero de 2014 y el 31 de octubre de 2015. La metodología utilizada en la recolección y análisis de datos es la descrita en documento publicado recientemente por el $\mathrm{GCRB}^{5}$, en concordancia con recomendaciones de SHEA/HICPAC $2008^{6}$. Se incluyeron los Servicios de UCI adultos (quirúrgico, médico, médico-quirúrgico o generales) y pediátricos. No se incluyeron intermedios, unidades de neonatología, unidad coronaria o UCI quemados. Un mismo hospital podía reportar datos de UCI pediátrica y de adultos, en tanto que la información fuera entregada por separado.

Para el numerador se solicitó información del número de aislados de cada una de las siete bacterias multiresistentes a vigilar: $S$. aureus resistente a oxacilina (meticilina) (SARM), Enterococcus resistente a vancomicina (ERV), Escherichia coli productora de BLEE, Klebsiella pneumoniae productora de BLEE, enterobacterias resistentes a carbapenémicos (ERC), Pseudomonas aeruginosa resistente a carbapenémicos y Acinetobacter baumannii resistente a carbapenémicos, definidos de acuerdo a los criterios de CLSI 20157. Los aislados en el numerador se consideraron bajo los siguientes criterios: (A) Sólo el primer aislado de cada bacteria con el patrón de resistencia mencionado por paciente (si un paciente presentara más de un cultivo positivo para la misma bacteria en menos de seis meses, sólo se consideró el primero); (B) Se consideraron cultivos obtenidos de muestras clínicas que pudieran representar tanto infección como colonización. Se excluyen resultados de cultivos obtenidos con fines de vigilancia epidemiológica; (C) Un mismo paciente puede tener más de una bacteria multi-resistente. Para el denominador se solicitó información del número de díascama ocupados correspondiente a cada mes del período en estudio, para cada Unidad de Cuidados Intensivos. Con la información entregada se realizó el siguiente cálculo para cada bacteria multi-resistente a vigilar: ( $\mathrm{N}^{\mathrm{o}}$ aislados/ días cama ocupados) x 1.000, obteniéndose el indicador de incidencia, expresado x 1.000 pacientes-día, tanto para UCI de adultos como pediátricos de cada hospital participante. Además, se realizó un consolidado nacional con mediana, percentiles $25-75$ e intervalo entre valor mínimo y máximo de incidencia para cada bacteria. Para evaluar diferencias de incidencia entre UCI de adultos y UCI pediátrica, se utilizó la prueba de Mann-Whitney para muestras independientes. Buscando proteger la confidencialidad de los datos y tal como se ha realizado en publicaciones anteriores, a cada hospital se le asignó un número que conocía sólo el equipo investigador y el responsable de cada centro.

\section{Resultados}

Se recibió información de 22 hospitales. De ellos, 14 fueron centros públicos, cuatro privados, dos institucionales y dos universitarios. Reportaron datos de 20 unidades de cuidados intensivos de adulto y nueve de pediatría. La mediana de camas de los hospitales fue de 348, mientras que la mediana de camas de UCI adulto fue de 12 y de pediatría de 7 (Tabla 1). Para cada bacteria multiresistente se describe la mediana de incidencia, percentil 25 , percentil 75 , mínimo y máximo, tanto para unidades de cuidados intensivos de adulto, como pediátricas (Tabla 2; Figuras 1 y 2).

Respecto a cocáceas grampositivas se analizaron SARM y ERV. S. aureus resistente a oxacilina tuvo alta incidencia alcanzando mediana de $3,85 \times 1.000$ pacientes-día en unidades de cuidados intensivos de adultos (UCI-A), ocupando el segundo lugar de incidencia. También se caracterizó por tener amplia dispersión entre hospitales (intervalo 0,71-12,66 x 1.000 pacientes-día). En unidades de cuidados intensivos pediátricos (UCI-P) la incidencia fue significativamente menor que en UCI-A, teniendo $75 \%$ de los hospitales una incidencia menor a 1 x 1.000 pacientes-día. Respecto a ERV, comparado con otras bacterias multi-resistentes, fue de baja incidencia con mediana de $1,56 \times 1.000$ pacientes-día y $47 \%$ de los hospitales con incidencia $\leq 1 \times 1.000$. La dispersión entre los hospitales fue menor a la observada en otras bacterias, con intervalo entre 0-6,17 x 1.000 pacientes-día. En UCI$\mathrm{P}$ la incidencia fue significativamente inferior, con tres hospitales que no tuvieron aislados en los 22 meses de estudio, y el resto con incidencias que no alcanzaron $1 \mathrm{x}$ 1.000 pacientes-día.

En relación a enterobacterias, se analizaron $E$. coli BLEE, K. pneumoniae BLEE y ERC. Respecto a $E$. coli BLEE se observó que en UCI-A la incidencia era moderada con mediana de 2,12 x 1.000 pacientes-día. Fue la bacteria que tuvo la menor dispersión entre los centros, con intervalo entre 0,76 y 5,5 . En UCI-P la incidencia fue significativamente menor, $78 \%$ de los hospitales tuvieron incidencia $<1 \times 1.000$ pacientes-día. En tanto K. pneumoniae BLEE fue, de las siete bacterias estudiadas, la que 


\begin{tabular}{|c|c|c|c|c|c|c|c|c|c|c|c|c|}
\hline $\mathrm{n}$ & $\begin{array}{l}\text { Tipo de } \\
\text { hospital }\end{array}$ & $\begin{array}{c}\mathbf{n} \\
\text { camas }\end{array}$ & $\begin{array}{c}\text { Tipo } \\
\text { de UCI }\end{array}$ & $\begin{array}{c}n \\
\text { camas }\end{array}$ & $\begin{array}{l}\text { SARM } \\
\text { (n) }\end{array}$ & $\begin{array}{l}\text { ERV } \\
\text { (n) }\end{array}$ & $\begin{array}{c}\text { E. coli } \\
\operatorname{BLEE~(+)~(n)~}\end{array}$ & $\begin{array}{c}\text { KLPN } \\
\text { BLEE (n) }\end{array}$ & $\operatorname{ERC}(n)$ & $\begin{array}{l}\text { PSAE } \\
\text { (n) }\end{array}$ & $\begin{array}{c}\text { ACBA } \\
\text { (n) }\end{array}$ & $\begin{array}{l}\text { n días } \\
\text { cama }\end{array}$ \\
\hline 1 & Público & $N / R$ & Adulto & $N / R$ & 12 & 4 & 4 & 25 & 5 & 9 & 2 & 5.293 \\
\hline 2 & Público & $N / R$ & Adulto & $N / R$ & 9 & 12 & 11 & 29 & 21 & 15 & 3 & 3.748 \\
\hline 3 & Público & $N / R$ & Pediátrica & $N / R$ & 1 & 0 & 1 & 5 & 6 & 1 & 0 & 3.053 \\
\hline 4 & Privado & 145 & Adulto & 24 & 6 & 2 & 13 & 16 & 0 & 6 & 0 & 8.467 \\
\hline 5 & Institucional & 362 & Adulto & 6 & 21 & 7 & 5 & 7 & 13 & 15 & 12 & 3.065 \\
\hline 6 & Institucional & 204 & Adulto & 8 & 9 & 2 & 9 & 11 & 5 & 11 & 3 & 3.360 \\
\hline 7 & Universitario & $N / R$ & Adulto & $N / R$ & 20 & 42 & 23 & 26 & 24 & 55 & 9 & 6.809 \\
\hline 11 & Público & 210 & Pediátrico & 45 & 8 & 2 & 6 & 27 & 6 & 6 & 0 & 19.317 \\
\hline 12 & Público & 536 & Adulto & 11 & 77 & 31 & 38 & 96 & 60 & 104 & 81 & 6.913 \\
\hline 13 & Público & 593 & Adulto & 17 & 69 & 32 & 23 & 75 & 50 & 59 & 87 & 10.648 \\
\hline 14 & Público & $N / R$ & Adulto & 12 & 44 & 3 & 8 & 28 & 0 & 13 & 23 & 7.507 \\
\hline 15 & Universitario & $N / R$ & Adulto & $N / R$ & 17 & $N / R$ & 33 & 28 & 45 & 30 & 12 & 17.529 \\
\hline 16 & Público & $N / R$ & Adulto & $N / R$ & 39 & 21 & 10 & 31 & 24 & 46 & 28 & 6.345 \\
\hline 23 & Público & 126 & Pediátrico & 10 & 12 & 0 & 6 & 23 & 8 & 18 & 2 & 7.272 \\
\hline 25 & Público & 378 & Adulto & 16 & 82 & 10 & 12 & 65 & 33 & 28 & 76 & 9.668 \\
\hline 26 & Público & 334 & Adulto & 6 & 13 & 0 & 8 & 4 & 0 & 4 & 6 & 3.313 \\
\hline 27 & Privado & 420 & Adulto & 12 & 23 & 20 & $N / R$ & $N / R$ & $N / R$ & 61 & 5 & 6.090 \\
\hline 29 & Público & $N / R$ & Adulto & $N / R$ & 69 & 32 & 23 & 75 & 50 & 59 & 87 & 10.648 \\
\hline 30 & Público & 511 & Adulto & 11 & 19 & 4 & 7 & 36 & 10 & 12 & 6 & 6.182 \\
\hline 31 & Privado & 332 & Adulto & 32 & 75 & 28 & 59 & 58 & 40 & 98 & 34 & 40.873 \\
\hline 34 & Privado & $N / R$ & Adulto & $N / R$ & 28 & 22 & 30 & 32 & 25 & 84 & 4 & 14.129 \\
\hline 38 & Público & 200 & Adulto & 4 & 23 & 2 & 17 & 36 & 2 & 17 & 2 & 5.676 \\
\hline 42 & Público & 511 & Pediátrica & 4 & 1 & 1 & 1 & 6 & 3 & 4 & 0 & 1.674 \\
\hline 44 & Público & 593 & Pediátrica & 9 & 13 & 1 & 10 & 9 & 5 & 17 & 3 & 3.321 \\
\hline 47 & Público & 487 & Adulto & 20 & 65 & 15 & 21 & 41 & 0 & 72 & $N / R$ & 5.136 \\
\hline 50 & Público & $N / R$ & Pediátrica & 7 & 1 & 1 & 6 & 17 & 0 & 6 & 1 & 3.737 \\
\hline 51 & Público & 378 & Pediátrica & 6 & 1 & 3 & 0 & 0 & 1 & 4 & 2 & 3.309 \\
\hline 52 & Universitario & $N / R$ & Pediátrico & $N / R$ & 6 & $N / R$ & 4 & 10 & $N / R$ & 5 & 0 & 8.649 \\
\hline 53 & Público & 487 & Pediátrico & 6 & 0 & 0 & 1 & 10 & 0 & 13 & 0 & 1.406 \\
\hline
\end{tabular}

tiene la mayor densidad de incidencia en Chile, tanto en UCI-A como en UCI-P, sin diferencia estadísticamente significativa entre ellas. En UCI-A alcanza alcanzó una mediana de 4,72, con un intervalo entre mínimo y máximo considerable (1,21-13,89). En UCI-P alcanza mediana de 2,71 y la gran mayoría tuvo incidencias $>1 \times 1.000$ pacientes-día, lo que fue considerablemente mayor a lo observado para otras bacterias en UCI-P. También tuvo un amplio intervalo de dispersión, que va entre $0,0-7,11$ x 1.000 pacientes-día. Respecto a ERC, alcanzó una mediana de 1,77 , pero la incidencia fue variable con un intervalo entre 0,0-8,68 x 1.000 pacientes-día. En pediatría alcanzó una incidencia menor, pero igualmente fue la tercera de mayor incidencia en este grupo.

Para $P$. aeruginosa resistente a carbapenémicos observamos que tuvo una alta incidencia en UCI-A con mediana de 3,64 x 1.000 pacientes-día (tercer lugar en incidencia) con amplio intervalo de dispersión entre 


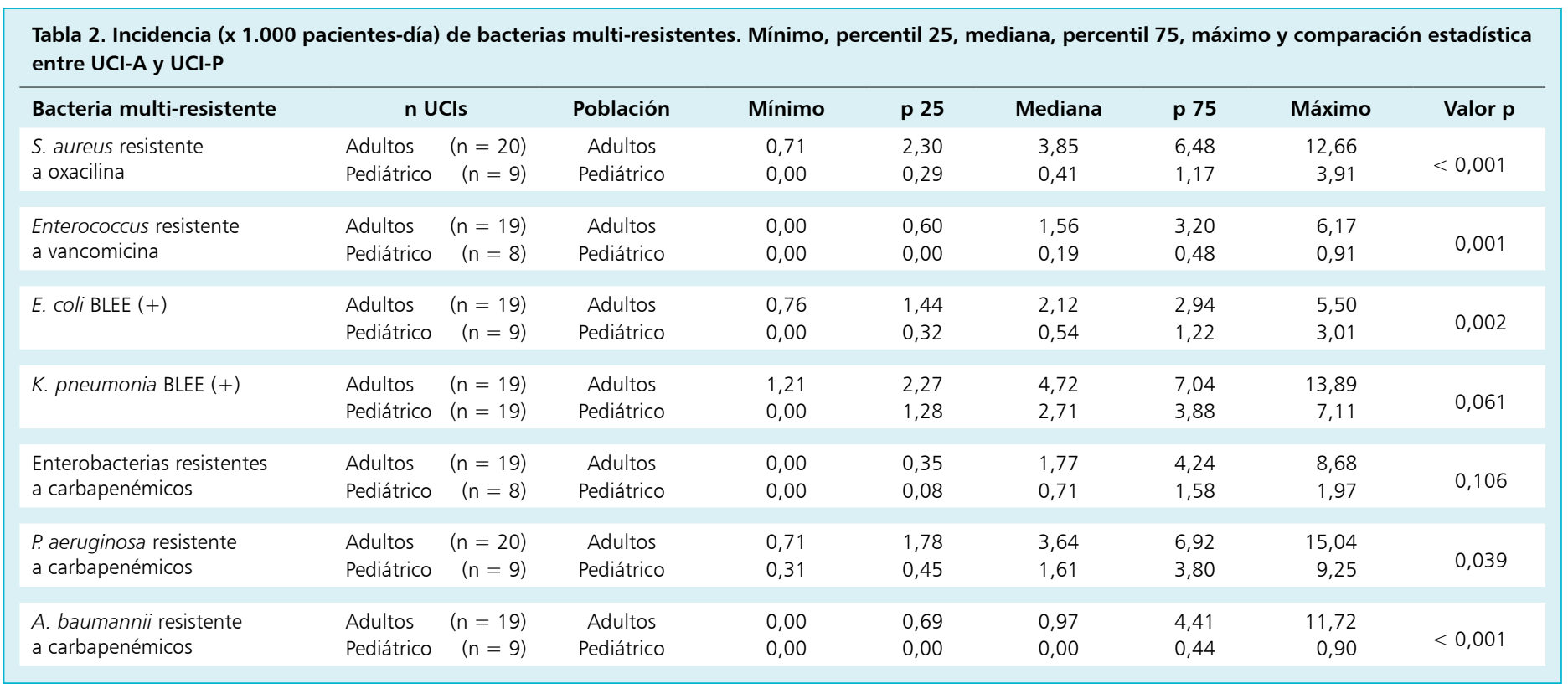

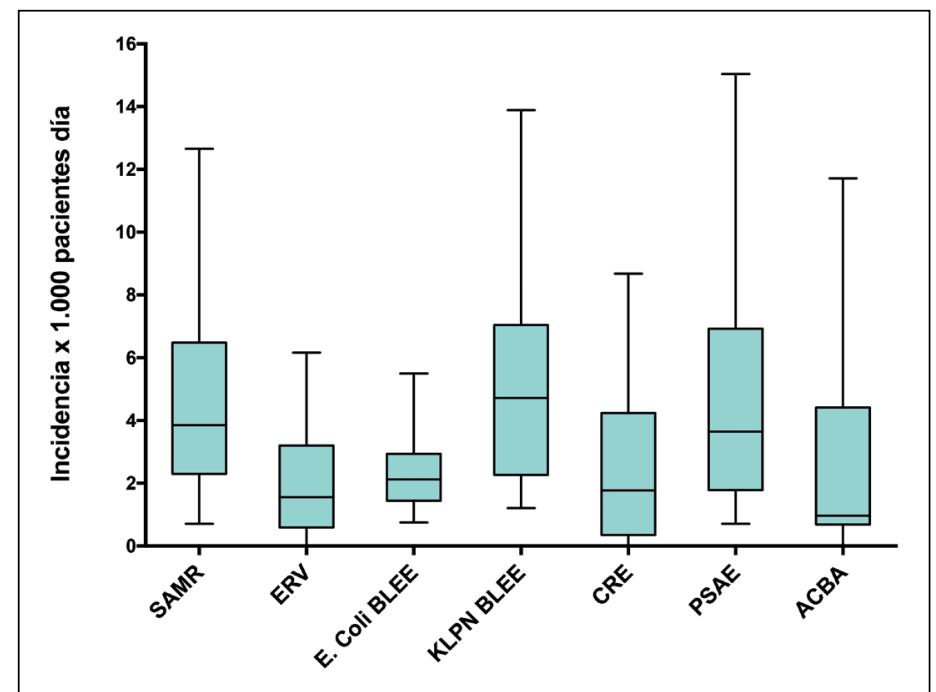

Figura 1. Incidencia (x 1.000 pacientes-día) de bacterias multi-resistentes en 20 unidades de cuidado intensivo de adulto en el período enero de 2014-octubre de 2015. Mediana, p25-p75 e intervalo mínimo-máximo. SARM $=$ S. aureus resistente a oxacilina (meticilina), $\mathrm{ERV}=$ Enterococcus resistente a vancomicina, $E$. coli $\mathrm{BLEE}=E$. coli productora de BLEE, $\mathrm{KLPN} B L E E=K$. pneumoniae productora de BLEE, $\mathrm{ERC}=\mathrm{En}$ terobacterias resistentes a carbapenémicos, PSAE = Pseudomonas aeruginosa resistente a carbapenémicos, ACBA = Acinetobacter baumannii resistente a carbapenémicos.

0,71-15,04 x 1.000 pacientes-día. En UCI-P también la incidencia fue alta, la mayor después de $K$. pneumoniae productora de BLEE, alcanzando mediana de $1,61 \times 1.000$ pacientes-día, nuevamente con amplio intervalo de dispersión $(0,31-9,25)$. Por último A. baumannii resistente a carbapenémicos, de las bacterias estudiadas, registró la menor incidencia tanto en UCI-A (mediana de $0,97 \times 1.000$ pacientesdía) como en UCI-P (mediana de 0,0). Además, en UCI-A fue una de

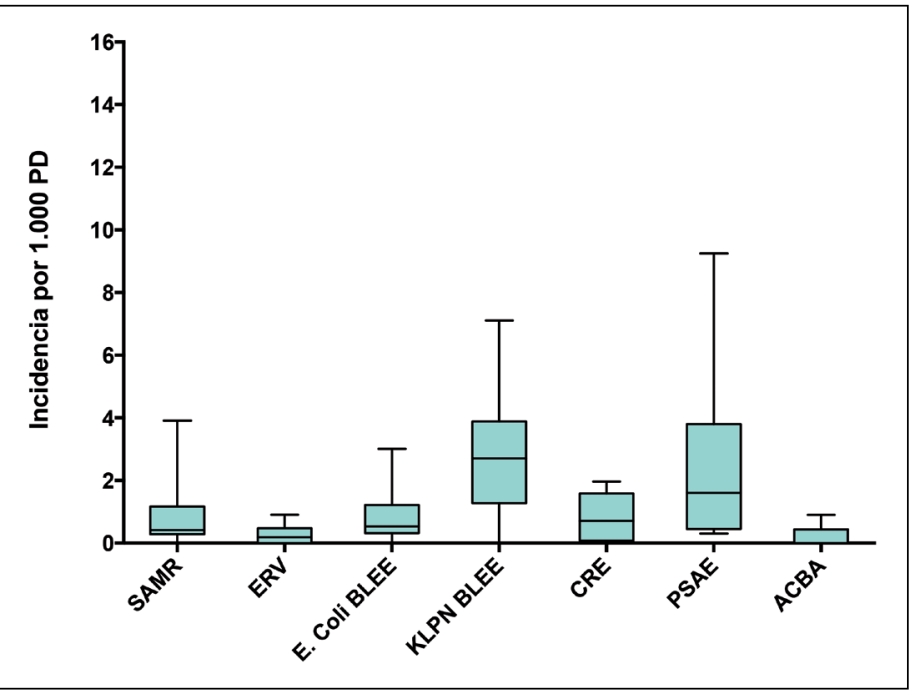

Figura 2. Incidencia (x 1.000 pacientes-día) de bacterias multi-resistentes en 9 unidades de cuidado intensivo pediátrico en el período enero de 2014-octubre de 2015. Mediana, p25-p75 e intervalo mínimo-máximo. SARM = S. aureus resistente a oxacilina, $\mathrm{ERV}=$ Enterococcus resistente a vancomicina, $\mathrm{KLPN} B L E E=K$. pneumoniae productora de $\mathrm{BLEE}, \mathrm{ERC}=$ Enterobacterias resistentes a carbapenémicos, PSAE $=$ Pseudomonas aeruginosa resistente a carbapenémicos, $\mathrm{ACBA}=$ Acinetobacter baumannii resistente a carbapenémicos.

las bacterias con el más amplio intervalo de dispersión entre hospitales alcanzando incidencias tan altas como 11,72 x 1.000 pacientes-día, pero por otra parte la mitad de los hospitales estudiados tuvo incidencia $<1$ x 1.000 pacientes-día, siendo de relevancia epidemiológica sólo en algunos hospitales. En UCI-P la incidencia fue inferior, con cinco hospitales que no tuvieron casos en los 22 meses del registro y el resto con incidencia menor a 1 x 1.000 pacientes-día. 


\section{Discusión}

Los datos aquí reportados corresponden al primer estudio de incidencia de bacterias multi-resistentes en UCI del país y, en nuestro conocimiento, no hay datos similares publicados en la región. Previamente el GCRB ha publicado la información susceptibilidad, expresado como el porcentaje de susceptibilidad/resistencia a distintos antimicrobianos entre los aislados de bacterias epidemiológicamente relevantes de un número significativo de hospitales chilenos ${ }^{3,4}$. Sin embargo, conocer la incidencia de estas bacterias en UCI permite precisar la magnitud del problema de resistencia para cada centro y establecer comparaciones entre ellos. Esto se ve reflejado al comparar los datos de resistencia bacteriana de 2012 publicados por el GCRB con nuestros resultados. En el estudio previo, el porcentaje de resistencia de E. faecium a vancomicina en UPC adulto alcanzó a $86 \%$. Sin embargo, en nuestro estudio se observa que es de baja incidencia ocupando el quinto lugar de las seis bacterias estudiadas, representando un problema epidemiológico sólo en algunos hospitales. Lo mismo ocurre con A. baumannii cuya resistencia a carbapenémicos en UCI es superior a $75 \%$ pero en términos de incidencia fue la de menor incidencia entre las bacterias estudiadas tanto en UCI de adultos como pediátrica. Por otra parte, en el caso de $P$. aeruginosa, la resistencia a carbapenémicos en UCI pediátrica alcanza $22-27 \%$, pero respecto a incidencia ocupa el segundo lugar en este grupo.

Los resultados de este estudio concluyen que la incidencia de bacterias multi-resistentes es significativamente mayor en hospitales de adulto en comparación con hospitales pediátricos, a excepción de $K$. pneumoniae productora de BLEE y enterobacterias resistentes a carbapenémicos en que, si bien la incidencia es mayor en UCI de adulto, no mostraron diferencias estadísticamente significativas. En el caso de A. baumannii y en menor medida también en SARM y $P$. aeruginosa resistente a carbapenémicos, se observa que la incidencia se concentra en algunos hospitales mientras que otros mantienen incidencias bajas, constituyendo un problema epidemiológico relevante sólo en algunas instituciones. Por otra parte, $E$. coli productora de BLEE tiene incidencia más bien estable, con poca dispersión en los distintos centros. Respecto a enterobacterias resistentes a carbapenémicos, siendo ésta una situación nueva para nuestros hospitales, se observa que es un problema transversal que afecta a $79 \%$ de las UCI-A estudiadas, aunque con incidencia moderada.

Experiencias similares en otras publicaciones son escasas. La gran mayoría de los estudios reportan porcentaje de resistencia frente a distintos antimicrobianos en los aislados de los laboratorios. Estudios de incidencia con metodología similar al nuestro se han realizado, pero en general enfocados en una bacteria multi-resistente en particular o una patología puntual y además, considerando a todos los pacientes hospitalizados, sin separar los datos de pacientes críticos, lo que baja considerablemente la incidencia y lo hace poco comparable a nuestros resulta$\operatorname{dos}^{8-10}$. Por otra parte, la mayoría son realizados en un solo centro y existen pocos estudios multicéntricos con una red de colaboración que entregue datos a nivel nacional como éste. Un reporte similar al aquí presentado con datos obtenidos también a través de una red colaborativa fue realizado en Alemania ${ }^{11}$. Incluyó 341 UCIs de 247 hospitales con resultados de incidencia considerablemente más bajos: 0,57 x 1.000 pacientes-día (PD) para SARM, 0,49 x 1.000 PD para ERV, 1,32 x 1.000 PD para gramnegativos multi-resistentes y $0,3 \times 1.000 \mathrm{PD}$ para bacterias resistentes a carbapenémicos. Otro estudio multicéntrico de vigilancia en Francia ${ }^{12}$ (933 hospitales) reportó la incidencia de SARM en UCI en 1,14 x 1.000 $\mathrm{PD}$, mientras que en enterobacterias productoras de BLEE fue de 1,63 x $1.000 \mathrm{PD}$, predominando E. coli por sobre $K$. pneumoniae, a diferencia de nuestros resultados.

Existen aún menos datos reportados de población pediátrica, que sería muy interesante de comparar, considerando que en nuestro estudio el comportamiento fue muy distinto al de población adulta, con incidencia significativamente menor en la mayoría de las bacterias estudiadas, pero además con distinta importancia relativa de ciertas bacterias como por ejemplo SARM, de mucha relevancia en población adulta, pero de baja incidencia en pediatría.

Una de las limitaciones que podemos reconocer en esta metodología es que la información obtenida se basa en auto-reporte voluntario de cada institución, lo que hace más difícil evaluar precisión de los datos. Por otra parte, si bien el número de hospitales incluidos es representativo, podrían incluirse aún más.

Este estudio provee información muy relevante de la magnitud del problema de resistencia bacteriana en UCI en el país. Es la primera vez que se obtiene esta información en Chile con un número muy significativo de unidades de cuidado intensivo participantes, tanto de adultos como pediátricos y sin datos publicados al respecto en América Latina. Conocer esta información permite comparar resultados locales con el universo de hospitales del país, permitiendo generar medidas orientadas al control y realizar un seguimiento en el tiempo. A partir de esta información, se evaluarán retrospectivamente datos de años anteriores para observar evolución. Además, se podría estudiar la relación que existe entre incidencia de bacterias multi-resistentes y el consumo de antimicrobianos de cada hospital. A futuro, se podría implementar vigilancia anual de densidad de incidencia de estas bacterias, para permitir seguimiento de la información en el tiempo y para tener una mejor evaluación de la resistencia bacteriana en nuestros hospitales y poder 
guiar los esfuerzos tanto a nivel local como a nivel país, de las medidas de control de transmisión de bacterias en el ambiente hospitalario y del uso de antimicrobianos.

Agradecimientos. Agradecemos muy especialmente a todos los hospitales que enviaron información y permitieron realizar este trabajo.

\section{Resumen}

Introducción: La vigilancia de incidencia de bacterias multi-resistentes es un indicador que permite estimar mejor la magnitud de la resistencia bacteriana en los servicios hospitalarios. Objetivo: Evaluar la incidencia de bacterias multi-resistentes relevantes en unidades de cuidados intensivos del país y establecer las diferencias entre población adulta y pediátrica. Metodología: Se solicitó a los hospitales participantes información del número de aislados de siete bacterias multi-resistentes epidemiológicamente relevantes de unidades de cuidados intensivos (UCI) de adulto y pediátrico entre enero de 2014 y octubre de 2015, y el número de días-cama ocupados en dichas unidades en el mismo período. Con estos datos se calculó incidencia por 1.000 pacientes-día para cada unidad. Resultados: Se recibió información de 20 UCI adultos y 9 UCI pediátricas. En UCI adultos las bacterias de mayor incidencia fueron $K$. pneumoniae productora de BLEE $[4,72$ x 1.000 días cama $(1,21-13,89)]$ y $S$. aureus resistente a oxacilina $[3,85(0,71-12,66)]$. En pediatría la incidencia fue menor, destacando $K$. pneumoniae productora de BLEE $[2,71(0-7,11)]$ y $P$. aeruginosa resistente a carbapenémicos $[1,61(0,31-9,25)]$. Conclusión: Se observan importantes diferencias entre los distintos hospitales en la incidencia de las bacterias estudiadas. La incidencia de bacterias multi-resistentes en UCI de adultos es significativamente mayor que en UCI pediátrica para la mayoría de las bacterias estudiadas.

\section{Referencias bibliográficas}

1.- Carvajal C, Versporten A, Severino N, Cifuentes M, Silva F, Rojas A, et al. The global point prevalence survey of antimicrobial consumption and resistance (Global-PPS): First results of antimicrobial prescribing in 12 Chilean hospitals. En: 26th European Congress of Clinical Microbiology and Infectious Diseases. 9-12 April 2016, Amsterdam, Netherlands.

2.- Domínguez I, Rosales R, Cabello A, Bavestrello L, Labarca J. Evaluación del consumo de antimicrobianos en 15 hospitales chilenos: Resultados de un trabajo colaborativo, 2013. Rev Chilena Infectol 2016; 33 (3): 307 12.

3.- Silva F, Cifuentes M, Pinto M E. Resultados de la vigilancia de susceptibilidad antimicrobiana en Chile: Consolidando una red. Rev Chilena Infectol 2011; 28 (1): 19-27.

4.- Cifuentes M, Silva F, García P, Bello H, Briceno I, Calvo M, et al. Susceptibilidad antimicrobiana en Chile 2012. Rev Chilena Infectol 2013; 31 (2): 123-30.
5.- Cifuentes M, Silva F, Arancibia J M, Rosales R, Ajenjo M C, Riedel G, et al. Grupo Colaborativo de Resistencia Bacteriana, Chile: recomendaciones 2014 para el control de la resistencia bacteriana. Rev Chilena Infectol 2015; 32 (3): 305-18.

6.- Cohen A L, Calfee D, Fridkin S K, Huang S S, Jernigan J A, Lautenbach E, et al. Recommendations for metrics for multidrugresistant organisms in healthcare settings: SHEA/HICPAC Position Paper. Infect Control Hosp Epidemiol 2008; 29: 901-13.

7.- CLSI. Performance Standards for Antimicrobial Susceptibility Testing: Twenty-Fifth Informational Supplement. CLSI document M100-S25. Wayne, PA: Clinical and Laboratory Standards Institute 2015.

8.- Willemsen I, Elberts S, Verhulst C, Rijnsburger M, Filius M, Savelkoul P, et al. Highly resistant gram-negative microorganisms: incidence density and occurrence of nosocomial transmission (TRIANGLe study). Infect Control Hosp Epidemiol 2011; 32 (4): 333-41.
9.- Arnaud I, Maugat S, Jarlier V, Astagneau P. Ongoing increasing temporal and geographical trends of the incidence of extended-spectrum beta-lactamase-producing Enterobacteriaceae infections in France, 2009 to 2013. Euro Surveill 2015; 20 (36).

10.- Freixas N, Sopena N, Limón E, Bella F. Surveillance of methicillin-resistant Staphylococcus aureus (MRSA) in acute care hospitals. Results of the VINCat Program (2008-2010). Enferm Infecc Microbiol Clin 2012; 30 (Supl 3): 39-42.

11.- Maechler F, Peña L A, Schröder C, Geffers C, Behnke M, Gastmeier P. Prevalence of carbapenem resistant organisms and other gramnegative MDRO in German ICUs: First results from the National Nosocomial Infection Surveillance System (KISS). Infection 2015; 43: 163-8.

12.- Carbonne A, Arnaud I, Maugat S, Marty N, Dumartin C, Bertrand X, et al. National multidrug-resistant bacteria (MDRB) surveillance in France Through the RAISIN Network: a 9 years experience. J Antimicrob Chemother 2013; 68: 954-9. 